\title{
HOPFIAN AND CO-HOPFIAN OBJECTS
}

\author{
K. VARADARAJAN ${ }^{ \pm}$
}

\begin{abstract}
The aim of the present paper is to study Hopfian and Co-Hopfian objects in categories like the category of rings, the module categories $A$-mod and mod- $A$ for any ring $A$. Using Stone's representation theorem any Boolean ring can be regarded as the ring $A$ of clopen subsets of a compact Hausdorff totally disconnected space $X$. It turns out that the Boolean ring $A$ will be Hopfan (resp. co-Hopfian) if and only if the space $X$ is co-Hopfian (resp. Hopfian) in the category Top. For any compact Hausdorf space $X$ let $C_{R}(X)$ (resp. $C_{C}(X)$ ) denote the $\mathcal{R}$ (resp. $C$ )-algebra of real (resp. complex) valued continuous functions on $X$. Using Gelfand's represertation theorem we will prove that $C_{\mathcal{R}}(X)\left(C_{C}(X)\right)$ is Ilopnan (respectively co-Hopfian) as an $\mathcal{R}(C)$ - algebra if and only if $X$ is co-Hopfian (respectively Hopfian) as an object of Top. We also study Hopfian and co-Hopfian compact topological manifolds.
\end{abstract}

\section{Introduction}

The notion of a Hopfian group [4] is by now classical. Throughout the present paper the rings $A$ we consider are associative rings with an identity element $I_{A} \neq 0$. Any subring $B$ of $A$ is required to satisfy the condition that $1_{B}=1_{A}$. All the modules considered are unitary modules. $A$-mod (resp. $\bmod -A$ ) will denote the caterogy of left (resp. right) $A$-modules. In [12] V.A. Hiremath has introduced the concept of Hopficity for a ring $A$ regarded as a ring and also for any $M \epsilon A$ mod. We will show in the present paper that $A$ is Hopfian in $A$-mod if and only if it is Hopfian in mod- $A$ (Theorem 1.3). When these two equivalent conditions are satisfied we will simply say that $A$ is Hopfan as a module. There are obvious dual notions of $A$ being co-Hopfan respectively as a ring, as an object in $A$-mod and as an object in mod- $A$. We obtain a necessary and sufficient condition for $A$ to be co-Hopfian in $A$-mod (Proposition 1.4). Unlike the Hopfian case, by means of a specific

\footnotetext{
${ }^{1}$ Research done while the author was partially supported by NSERC grant A8225.
} 
example we show that co-Hopficity is not left-right symmetric. Also, we will give examples to show that $A$ being Hopfian (resp. co-Hopfian) as a ring and $A$ being Hopfian as a module (resp. co-Hopfian in $A$-mod or $\bmod -A$ ) are independent of each other. As an immediate consequence of our necessary and sufficient condition it will follow that a not necessarily commutative integral domain $A$ is co-Hopfian in $A$-mod as well as mod- $A$ if and only if $A$ is a skew-field.

It is a well-known result that any noetherian $M \epsilon A$-mod is Hopfian in $A$-mod and that any artinian $M \epsilon A$-mod is co-Hopfian ([17, page 42]). Arguments used in proving this result will show that any ring $A$ with a.c.c. on two sided ideals is Hopfian as a ring and any ring $A$ with d.c.c on subrings is co-Hopfian as a ring. In particular any left noetherian (herce any left artinian) ring $A$ is Hopfian as a ring. Easy examples can be given to show that even ficlds need not be co-Hopfian as rings. Similar to the result that any left artinian ring is left noctherian we have the result that any ring $A$ which is co-Hopfian in $A$-mod is automatically Hopfian in $A$-mod, hence also Hopfian in mod-A (Proposition 1.10). Let $n$ be any integer $\geq 1$. It is easy to prove the following implications:

a) $M_{n}(A)$ Hopfan (resp. co-Hopfian) as a ring $\Rightarrow A$ Hopfian (resp. co-Hopfian) as a ring.

b) $M_{n}(A)$ Hopfian (resp. co-Hopfian) in $M_{n}(A)$-rnod $\Rightarrow A$ Hopfian (resp. co-Hopfian) in $A$-mod.

The analogue of Hilbcrt's basis theorem is valid for Hopficity, namely $M \in A$-mod is Hopfian if and only if $M[X]$ is Hoptian in $A[X]$-mod, where $X$ is an indeterminate over $A$. This and the analogous result for $M[[X]]$ in $A[[X]\}$-mod are proved in Section 2 of the present paper (Theorem 2.1). We do not know whether the analogous result is valid for $M\left[X, X^{-1}\right]$ in $A\left[X, X^{-1}\right]$-mod. For any non-zero $M \in A$-mod, it is easy to see that $M[X]$ (resp. $M[[X]]$ ) is not co-Hopfian in $A[X]$ (resp. $A[[X]]$ )-mod.

In Section 3 we are mainly concerned with the case when $A$ is commutative. For the results stated in the present paragraph it will be assumed that $A$ is a commutative ring. Then it is well-known [22], [24] that every f.g. (abbreviation for finitely generated) $A$-module is Hopfian. It can easily be shown that $M_{n}(A)$ is Hopfian in $M_{n}(A)-\bmod$ or $\bmod -M_{n}(A)$ for all integers $n \geq 1$. Our necessary and sufficient condition for $A$ to be co-Hopfian in $A$-mod (Theorem 1.3) implies that $A$ is co-Hopfian in $A-\bmod \Leftrightarrow A$ is its own total quotient ring. In this case we will prove that $M_{n}(A)$ is co-Hopfian in both $M_{n}(A)-\bmod$ and $\bmod -M_{n}(A)$. We will also prove that $A^{n}$ is co-Hopfian in $A$-mod for all $n \geq 1$. The proof of this will depend on an auxiliary result asserting that an $A$-homomorphism $f: A^{n} \rightarrow A^{n}$ is not injective if and only if det $f$ is a zero divisor in $A$, whatcuer be the commutative ring $A$ (kmmia 3.1). It is also well-known 
[2], [25] that every f.g A-module is co-Hopfian if and only if every prime ideal of $A$ is maximal. We will explicitly constract a commutative ring $A$ which is its own total quotient ring admitting a prime ideal which is not maximal. In particular this ring will satisfy the condition that $A^{n}$ is co-Hopfian in $A$-mod for cach integer $n \geq \mathrm{I}$ and there are f.g $A$-rnodulcs which are not co-Hopfian. The ring $A$ that we construct will have the following additional properties:

c) $A$ is not noetherian

d) $A$ does not have d.c.c for subrings

In [12] Hiremath shows that if the Boolean ring of clopen subsets of a compact Hausdorff totally disconnected space $X$ satisfics the condition that $A$ is Hopfian as a ring then $X$ is co-Hopfan as a topological space. He says he does not know whether the converse to this result is true. Actually we not only show that the converse is true but we also show that $A$ is co-Hopfan as a ring if and only if $X$ is Hopfian as a topological space. This is carried out in Section 4.

Let, $X$ denote a compact Hausdorff space and $C(X)$ denote either $C_{\mathcal{R}}(X)$ or $C_{\mathcal{C}}(X)$. We regard $C_{\mathcal{R}}(X)$ as an $\mathcal{R}$-algebra and $C_{\mathcal{C}}(X)$ as a $\mathcal{C}$-algebra and simply write "the algebra $C(X)$ ". Using Gelfand's representation theorem we show that $C(X)$ as an algebra is Hopfian (resp. co-Hopfian) if and only if $X$ is co-Hopfian (resp. Hopfian) in the category Top of topological spaces. (Theorem 5.3). Wo do not have any characterization of compact Hausdorff spaces which are Hopfian (resp. co-Hopfian). However it is an easy consequence of invariance of domain that compact topological manifolds without boundary are co-Hopfian. Among compact manifolds without boundary it can easily be shown that finite sets are the only Hophan objects. Among compact manifolds with a non-empty boundary there are no Hopfan or co-Hopfian objects. If $M$ is a compact manifold with boundary $\partial M$ then the pair $(M, \partial M)$ is a co-Hopfian object in the category Top ${ }^{2}$ of pairs of topological spaces.

We conclude our introduction by pointing out that Hilton, Roitberg etc., have studied epimorphisms and monomorphisms in the honotopy category and were led to investigating Hopfian and co-Hopfian objects in the homotopy category [10], [18]. Finally we wish to thank the referee for information on literature. In fact most of the material in Section 7 has been pointed out by the referce.

Acknowledgements. Part of this work was done while the author was visiting Centre de Recerca Matematica, Bellaterra in Spain. The author would like to thank Professor Castellet for crcating a very conducive atmosphere for research. Also while carrying out this research the author received support from NSERC grant A8225. 


\section{Hopfian and co-Hopfian rings and modules}

Throughout we will formulate our results in the category $A$-nod of left urital $A$-modules. There are obvious analogous results in the category $\bmod -A$ of unital right $A$-modules. We first fix our terminology and notation. For any $a \epsilon A, \ell_{A}(a)=\{b \epsilon A \mid b a=0\}$ and $r_{A}(a)=\{b \epsilon A \mid a b=0\}$. By a left (resp. right) zero divisor in $A$ we mean an element $a \neq 0$ in $A$ with $\ell_{A}(a) \neq 0$ (resp. $r_{A}(a) \neq 0$ ). An element $a \in A$ will be called a left (resp. right) unit if there exists an element $c \epsilon A$ with $c a=1$ (resp. $a c=1$ ). We call $a \in A$ left (resp. right) regular if $\ell_{A}(a)=0$ (resp. $r_{A}(a)=0$ ). It is trivial to see that any left (rcsp. right) zcro divisor is never a right (resp. left) unit. Also any left regular element $a$ which is a left. unit is automatically a two-sided unit.

Definition 1.1. $M \epsilon A$-mod is said to be Hopfian (resp. co-Hopfiar) if every surjective (resp. injective) homomorphism $f: M \rightarrow M$ is an isomorphisrn.

It is well-known that any noetherian (resp. artinian) module is Hopfian (resp. co-Hopfian) [17, Lemma 4, page 41].

Proposition 1.2. AtA-mod is Hopfian if and only if no left zero divisor in $A$ is a left unit in $A$. This is theorem 9 in [12]. Equivalently it is well-known and easy to see that $A \in A$-mod is Hopfian if and only if $A$ is directly finite (i.e. $x y=1 \Rightarrow y x=1$ ) [11].

Theorem 1.3. AtA-mod is Hopfian if and only if At mod-A is Hopfian.

Proof: Direct finiteness is clearly left right symmetric.

Proposition 1.4. AєA-mod is co-Hopfian if and only if every left regular element $a \in A$ is a tuo-sided unit.

Proof: Immediate consequence of the fact that injective homomorphisms $f: A \rightarrow A$ in $A$-mod are exactly given by $f(\lambda)=\lambda a$ with $a \in A$ left regular.

Examples 1.5. Consider the ring

$$
A=\left[\begin{array}{ll}
\mathcal{Z} / 2 \mathcal{Z} & \mathcal{Z} / 2 \mathcal{Z} \\
0 & \mathcal{Z}_{(2)}
\end{array}\right]
$$

where $\mathcal{Z}_{(2)}$ is the 2-localization of $\mathcal{Z}$, namely $\mathcal{Z}_{(2)}=\left\{\frac{m}{n} \epsilon Q \mid n\right.$ odd $\}$. The element $\left(\begin{array}{ll}1 & 1 \\ 0 & 2\end{array}\right) \epsilon A$ is easily checked to be right regular but not invertible in $A$. Hence $A$ is not co-Hopfian in $\bmod A$. 
(b) The only ring homomorphism of $\mathcal{Z}$ (resp. $\mathcal{Q}$ ) is the identity map. Hence $Z$ and $\mathcal{Q}$ are Hopfian and co-Hopfian as rings. While $Z$ is Hopfian in $Z$-mod, it is not co-Hopfian in $\mathcal{Z}$-mod. $Q$ is both Hopfian and co-Hopfian in $\mathcal{Q}$-mod (hence also in $Z$-mod).

(c) For any ring $A$ the unique ring homomorphism $\varphi: A[X] \rightarrow A[X]$ carrying $X$ to $X^{2}$ and satisfying $\varphi \mid A=I d_{A}$ is an injective ring homomorphism which is not surjective. (Here $X$ is an indeterminate over $A$ ). Thus $A[X]$ is not co-Hopfian as a ring, whatever be the ring $A$. A similar argument shows that $A\left[X, X^{-1}\right]$ and $A[[X]]$ are not co-Hopfian as rings.

(d) Let $A=K\left[\left(X_{\alpha}\right)\right]_{\alpha \epsilon J}$ over a commmtative ring $K$. Then $A$ is a commutative ring hence Hopfinn in $A$-mod. Let $\Theta: J \rightarrow J$ be a surjective map which is not bijective. Since $J$ is infinite such a map exists. The unique ring homomorphism $f: A \rightarrow A$ satisfying $f \mid K=I d_{K}$ and $f\left(X_{\alpha}\right)=X_{\Theta(\alpha)}$ is then a surjective ring homomorphism which is not an isomorphism. Thus $A$ is not Hopfian as a ring.

(e) Let. $K$ be a field and $L=K\left(\left(X_{\alpha}\right)_{\alpha \epsilon J}\right)$ the field of rational functions in an infinite number of indetcrminates. Any field is Hopfan as a ring. Thus $L$ is Hopfian as a ring. If $\Theta: J \rightarrow J$ is any injective map, there is a unique homomorphism $\varphi: L \rightarrow L$ of fields satisfying $\varphi\left(X_{\alpha}\right)=X_{\Theta(\alpha)}$ and $\varphi / K=I d_{K}$. If $\Theta$ is not bijective, then $\varphi$ is an injective ring homomorphism of $L$ in $L$ which is not surjective. Hence $L$ is not co-Hopfian as a ring. Since $L$ if a field from remarks $1.6(\mathrm{a})$ and (c) we sec that $L$ is both co-Hophan and Hopfian in $L$-mod.

(f) For any simple ring $A$ any ring homomorphism $f: A \rightarrow B$ is automatically injective. Hence every simple ring is Hopfian as a ring. From example (e) above we see that a simple ring (even a field) need not be co-Bopfan as a ring.

(g) Let $K$ be a field and $V$ an infinite dimensional vector space over $K$. Let $A=$ End $_{K} V$. There exist $K$-linear surjections $f: V \rightarrow V$ which are not injective. Choose such an $f$. Since $V \stackrel{f}{\rightarrow} V \rightarrow 0$ splits in $K$-rnod, $\exists$ a $K$-linear map $h: V \rightarrow V$ with $f \circ h=I d V$. This means $f$ is a right unit in $A$. Since ker $f \neq 0$ we can choose a. $g: V \rightarrow V$ with $g \neq 0$ and $g(V) \subset$ Ker $f$. Then $g \in A$ satisfies $f \circ g=0$. Thus $f$ is a right zero divisor in $A$ which is not a right unit in $A$. From proposition 1.4 we see that $A$ is not Hopfian in rnod- $A$ and hence also not in $A$-mod from theorem 1.3 . In case $V$ has countable dimension it follows from exercise 14.13, page 164 of [1] that there are only two non-zero ideals in $A=$ End $_{K} V$. Hence $A$ is Hopfian as a ring (see proposition 1.12). 
(h) If $A=K\left[\left(x_{\alpha}\right)_{\alpha c}\right]$ with $K$ any commutative ring and $J$ infinite, from 1.8(d) we see that $A$ is Hopfian in $A$-mod, but not Hopfian as a ring. When $K$ is a field and $V$ a countably infinite dimerisional vector space then $A=$ End $_{K} V$ is Hopfian as a ring but not Hopfian as an $A$-module. As already seen $Z$ is co-Hopfian as a ring but not as a $\mathcal{Z}$-module. When $K$ is a field, $A=K\left(\left(X_{\alpha}\right)_{\alpha \in J}\right)$ the field of rational functions in an infinite number of indeterminates is an example of a commutative ring which is co-Hopfian as a module but not co-Hopfian as a ring from $1.8(\mathrm{e})$.

\section{Proposition 1.9.}

(i) If $A$ is a ring satisfying a.c.c for two sided ideals then $A$ is Hopfan as a ring.

(ii) If $A$ is a ring satisfying d.c.c for subrings then $A$ is co-Hopfan as a ring.

The proof of this proposition is similar to that of lemma 4 , page 42 of $[17]$ and hence omitted.

Proposition 1.10. Let $A$ be a ring with the property that $A$ is coHopfian in $A$-mod. Then $A$ is automatically Hopfian in A-mod.

Proof: Let a be a left zero divisor in $A$. From proposition 1.2 we have only to show that a is not a left unit in $A$. On the contrary if $a$ is a left unit in $A$, there exists an element $c \epsilon A$ with $c a=1$. Then clearly $c$ is left regular. Since $A$ is co-Hopfian in $A$-mod, from proposition 1.4 we see that $c$ is a two sided unit in $A$. Then $c a=1$ implics that $a$ is the inverse of $c$ and hence $a$ is also a two sided unit. This contradicts the fact that $a$ is a left zero divisor.

Remarks 1.11. Hiremath [12] has already observed that a direct summand of any Hopfan module is Hopfian. The same observation is valid for co-Hopfian modules as well. He remarks that he does not know of any example of a Hopfian module with a submodule not Hopfian. Later in Section 3 we will construct such modules. $Q$ is Hopfian and coHopfian in $\mathcal{Z}$-mod, the quotients $\mathcal{Z}_{p_{\infty}}$ of $Q$ are not Hopfan in $\mathcal{Z}$-rnod. Later results in Section 3 will also show that quotients of co-Hopfian modules need not be co-Hopfian.

Proposition 1.12. Let $A$ be a ring and $n$ an integer $\geq 1$. Then

(i) $M_{n}(A)$ Hopfan (resp. co-Hopfian) as a ring $\Rightarrow$ A Hopfan (resp. co-Hopfian) as a ring.

(ii) $M_{n}(A)$ Hopfian (resp. co-Hopfian) in $M_{n}(A)-\bmod \Rightarrow A$ Hopfian (resp. co-Hopfan) in A-mod. 
(iii) $M_{n}(A)$ Hopfian (resp. co-Hopfian) in A-mod $\Rightarrow A$ Hopfian (resp. co-Hopfian) in A-mod.

Proof:

(i) is an immediate consequence of the observations that if $f: A \rightarrow A$ is a ring homomorphism, $M_{n}(f): M_{n}(A) \rightarrow M_{n}(A)$ defined in the obvious way is a ring homomorphism and that $M_{n}(f)$ is surjective (resp. injective) $\Leftrightarrow f$ is surjective (resp. injective).

(ii) similar to (i) above except for the observation that if $f: A \rightarrow A$ is a map in $A$-mod, then $M_{n}(f): M_{n}(A) \rightarrow M_{n}(A)$ is a map in $M_{n}(A)$-mod.

(iii) is immediate from the fact that $A$ is a direct summand of $M_{n}(A)$ in A-mod.

The converses for (ii), (iii) arc not true in general. Counter examples will be given in Section 7. But when $A$ is commutative for (ii), (iii) the converses are true and they will be proved in Secton 3 . We do not know whether the converse for (i) is true.

Given any non-zero $M \in A$-mod it is known that any infinite direct sum of copics of $M$ is neither Hopfian nor co-Hopfian in $A$-mod. Any such module will admit the module $N=\oplus_{n \geq 1} M_{n}$ as direct summand where $M_{n}=M$ for cach $n \geq 1$. The shift map $s_{+}$which carries the $n$th copy of $M$ to the $(n+1)^{s t}$ copy identically is an injective map which is not surjective. The shift map $s_{\text {- }}$ which maps the $(n+1)^{s t}$ copy to the $n^{\text {th }}$ copy ideritically for $n \geq 1$ and which maps the $1^{\text {st }}$ copy of $M$ to zero is a surjective map which is not injective. This fact will be made use of by us later in Section 3 for constructing a Hopfian module adnitting a nonHopfan submodule. An infinite direct sum of non-zero modules could very well be simultaneously Hopfian and co-Hopfiar. If $P$ denotes the set of all primes, $M=\oplus_{p \in} P(\mathcal{Z} / p \mathcal{Z})$ is easily seen to be sirnultaneously Hopfian and co-Hopfian in $\mathcal{Z}$-mod,

Proposition 1.13. Let $A[G]$ denote the group ring of a group $G$ over the ring $A$. If $A[G]$ is Hopfian (resp. co-Hopfian) as a ring then $A$ is Hopfian (resp. co-Hopfian) as a ring and $G$ is Hopfian (resp. co-Hopfian) as a group.

Proof: Let $f: A \rightarrow A$ be a homomorphism of rings and $\varphi: G \rightarrow G$ a homornorphism of groups. Then the map $\beta: A[G] \rightarrow A[G]$ defined by $\beta\left(\sum_{g \in C} a_{g} g\right)=\sum_{g \in G} f\left(a_{y}\right) \varphi(g)$ is a ring homomorphism. Also it is easily checked that $\beta$ is surjective (resp. injective) $\Leftrightarrow f$ and $\varphi$ are surjective (resp. injective). Proposition 1.13 is an easy consequence of these facts. 
Remarks 1.14. If $f: A \rightarrow A$ is a map in $A$-mod and $\varphi: G \rightarrow G$ is a group homomorphism it is in general not true that $\beta: A[G] \rightarrow A[G]$ defined $\beta\left(\sum_{g \in G} a_{g} g\right)=\sum_{g t G} f\left(a_{g}\right) \varphi(g)$ will be a map in $A[G] \cdot \bmod$. In case $\varphi=I d_{C}$ it is true that $\beta$ is a map in $A[G]$-mod. The analogue of 1.13 for module categories is not valid. If $A$ is any commutative ring and $G$ any abelian group, $A[G]$ is Hopfian in $A[G]$-mod. $G$ need not be Hopfian. However, the following can be proved.

Proposition 1.15. If $A[G]$ is Hopfian (resp. co-Hopfian) in $A \mid G]-$ mod, then A is Hopfian (resp. co-Hopfian) in A-mod.

Proposition 1.16. Let $\left\{A_{\alpha}\right\}_{\alpha \epsilon J}$ be any family of rings and $A=$ $\Pi_{\alpha \varepsilon, J} A_{\alpha}$ their direct product.

(i) A is Hopfan (resp. co-Hopfian) in $A$-mod $\Leftrightarrow$ each $A_{c x}$ is Hopfian (resp. co-Hopfian) in $A_{\alpha}-$ mod.

(ii) If $A$ is Hopfian (resp. co-Hopfian) as a ring then each $A_{\alpha x}$ is Hopfian (resp. co-Hopfian) as a ring.

Proof:

(i) is an immediate consequence of the fact that any map $f: A \rightarrow A$ in $A$-rnod is uniquely of the form $\Pi f_{\alpha}: \Pi A_{\alpha} \rightarrow \Pi A_{\alpha}$ with $f_{\alpha}$ : $A_{\alpha} \rightarrow A_{\alpha}$ a map in $A_{\alpha}$-mod and $f$ is surjective (resp. injective) $\Leftrightarrow$ cach $f_{\alpha}$ is surjective (resp. injective).

(ii) If $f_{\alpha}: A_{\alpha} \rightarrow A_{\alpha}$ is a ring homomorphism for each $\alpha \epsilon$, $J$, then $f=\Pi f_{\alpha}: \Pi A_{\alpha} \rightarrow \Pi A_{\alpha}$ is a ring homomorphism. Moreover $f$ is surjective (resp. injective) if and only if each $f_{\alpha}$ is surjective (resp. injective). (ii) is an immediate consequence of these facts.

Actually proposition 1.16(i) can be improved as follows:

Proposition 1.17. Let $\left\{A_{\alpha}\right\}_{\alpha \epsilon J}$ be any family of rings and $A=$ $\Pi_{\alpha \epsilon, J} A_{\alpha}$ their direct product. Let $M_{\alpha} \in A_{\alpha}$-mod for each $\alpha \epsilon_{J} J$. If $M=$ $\Pi_{\alpha \epsilon} J M_{\alpha}$ with $A$-action defined by a.m $=\left(a_{\alpha x} m_{\alpha}\right)_{c}$, whenever $a=\left(a_{\alpha x}\right)_{\alpha \in J}$ with $a_{\alpha} \in A_{1 x}$ and $m=\left(m_{\alpha}\right)_{\alpha \in I}$ with $m_{\alpha} \in M_{\alpha}$. Then $M$ is Hopfian (resp. co-Hopfian) in $A$-mod if and only if each $M_{\alpha}$ is Hopfan (resp. co-Hopfion) in $A_{\alpha}$-mod.

Again this is an immediate consequence of the fact that any map $f$ : $M \rightarrow M$ in $A$-mod is uniquely of the form $\Pi f_{\alpha}: \Pi M_{\alpha \alpha} \rightarrow \Pi M_{\alpha x}$ with $f_{\alpha}$; $M_{\alpha} \rightarrow M_{\alpha}$ a map in $A_{c \alpha}-$ mod.

\section{Hopficity of the modules $M[X], M[X] /\left(X^{n}\right)$ and $M[[X])$}

Given any $M \in A$-mod and an indeterminate $X$ over $A$ we define 
$M[X] \epsilon A[X]-\operatorname{rrod}, M[X] /\left(X^{n}\right) \epsilon A[X] /\left(X^{n}\right)-\bmod$ and $M[[X]\} \epsilon A[[X]]-\bmod$ as in Section 1 of $[23]$, where $A[X]$ denotes the polynomial ring, $A[X] /\left(X^{n}\right)$ for any integer $n \geq 1$ denotes the truncated polynomial ring and $A[[X]]$ the formal power series ring. The main result proved in this section is the following:

Theorem 2.1. Let $M \epsilon A$-mod. Then the folowing are equivalent.

(1) $M$ is Hopfian in A-mod.

(2) $M[X]$ is Hopfian in $A[X]$-mod.

(3) $M[X] /\left(X^{n}\right)$ is Hopfan in $A[X] /\left(X^{n}\right)$-mod.

(4) $M[[X]]$ is Hopfian in $A[[X]]-$ mod.

Proof: (2) $\Rightarrow(1)$. Let $f: M \rightarrow M$ be any surjectivc map in $A$ $\bmod$. Then $f[X]: M[X] \rightarrow M[X]$ defined by $f[X]\left(\sum_{j=0}^{k} a_{j} X^{j}\right)=$ $\sum_{j=0}^{k} f\left(a_{j}\right) X^{j}$ is a surjective map in $A[X]$-mod. Since $M[X]$ is Hopfian in $A[X]$-mod we sce that $f[X]$ is injective. This immediately yields the injectivity of $f$.

The proofs of $(3) \Rightarrow(1)$ and $(4) \Rightarrow(1)$ are similar and orritted.

(1) $\Rightarrow(2)$. Let $\varphi: M[X] \rightarrow M[X]$ be any surjective $A[X]$-hornorphism. Let $\theta=\varphi \mid M: M \rightarrow M[X]$. Then $\theta$ is an $A$-homomorphism: Morcover

$$
\varphi\left(\sum_{j=0}^{k} a_{j} X^{j}\right)=\sum_{j=0}^{k} X^{j} \theta\left(a_{j}\right) .
$$

For any $i \geq 0$ let $p_{i}: M[X] \rightarrow M$ be defined by

$$
p_{i}\left(\sum_{j=0}^{k} a_{j} X^{j}\right)= \begin{cases}a_{2} & \text { if } i \leq k \\ 0 & \text { if } i>k\end{cases}
$$

Then $p_{i}: M[X] \rightarrow M$ is a map in $A$-mod for each $i \geq 0$. Since $\varphi$ is surjective, given any ce $M$ there exist an element $\sum_{j=0}^{k} a_{j} X^{j} \in M[X]$ with $\varphi\left(\sum_{j=0}^{k} a_{j} X^{j}\right)=c$.

Using 4 , we sec that the "constant term" of $\theta\left(a_{0}\right)$ is $c$ or cquivalently $p_{0} \circ \theta\left(a_{0}\right)=c$. This shows that the map $p_{0} \circ \theta: M \rightarrow M$ is a surjective map in $A$-rnod. The Hopfan nature of $M$ in $A$-rnod implies that $p_{0} \circ \theta: M \rightarrow M$ is an isomorphism, in particular injective.

Our ain is to show that $\varphi: M[X] \rightarrow M[X]$ is injective. Let $\sum_{j=0}^{k} b_{j} X^{j} \in$ $M[X]$ satisfy $\varphi\left(\sum_{j=0}^{k} b_{j} X^{j}\right)=0$. Using 4 and observing that $\theta\left(b_{j}\right)=$ 
$\sum_{i \geq 0} p_{i} \circ \theta\left(b_{j}\right) X^{i}$ we see that $\varphi\left(\sum_{j=0}^{k} b_{j} X^{j}\right)=\sum_{j=0}^{k} d_{j} X^{j}+$ terms involving higher powers of $X$ where

$$
d_{j}=p_{j} \circ \theta\left(b_{0}\right)+p_{j-1} \circ \theta\left(b_{1}\right)+\ldots+p_{0} \circ \theta\left(b_{j}\right)
$$

for $0 \leq j \leq k$. Hence $\varphi\left(\sum_{j=0}^{k} b_{j} X^{j}\right)=0$ implises $b_{j}=0$ for $0 \leq j \leq k$. Writing these out we get the following system of cquations:

$$
\left.\begin{array}{c}
p_{0} \circ \theta\left(b_{0}\right)=0 \\
p_{1} \circ \theta\left(b_{0}\right)+p_{1} \circ \theta\left(b_{1}\right)=0 \\
p_{2} \circ \theta\left(b_{0}\right)+p_{1} \circ \theta\left(b_{1}\right)+p_{0} \circ \theta\left(b_{2}\right)=0 \\
\ldots \ldots \ldots \ldots \ldots \ldots \ldots \ldots \\
p_{k} \circ \theta\left(b_{0}\right)+p_{k-1} \circ \theta\left(b_{1}\right)+\ldots \ldots+p_{0} \circ \theta\left(b_{k}\right)=0
\end{array}\right\}
$$

We know that $p_{0} \circ \theta$ is injective. Hence from the first of these equations we get $b_{0}=0$. Substituting this in the second equation we get $p_{0} \circ \theta\left(b_{1}\right)=$ 0 , hence $b_{1}=0$. Now the third equation will yield $p_{0} \circ \theta\left(b_{2}\right)=0$, herce $b_{2}=0$. Proceeding thus we see that $b_{0}=b_{1}=\ldots=b_{k}=0$. Herce $\varphi$ is injective.

(1) $\Rightarrow(4)$. The proof is sirnilar to that of $(1) \Rightarrow(2)$. We will only indicate the changes needed. In the proof replace $M[X]$ by $M[[X]$, $\sum_{j=0}^{k} a_{j} X^{j}$ by $\sum_{j \geq 0} a_{j} X^{j}$, equation 4 by $\varphi\left(\sum_{j \geq 0} a_{j} X^{j}\right)=\sum_{j \geq 0} X^{j} \theta\left(a_{j}\right)$. The $p_{i}$ 's are defincd by $p_{i}\left(\sum_{j \geq 0} a_{j} X^{j}\right)=a_{i}$ for all $i \geq 0$. The calculation of $\varphi\left(\sum_{j \geq 0} b_{j} X^{j}\right)$ will now be $\varphi\left(\sum_{j \geq 0} b_{j} X^{j}\right)=\sum_{j \geq 0} d_{j} X^{j}$ where $d_{j}=p_{j} \circ \theta\left(b_{0}\right)+\ldots+p_{0} \circ \theta\left(b_{j}\right)$. Hence $\varphi\left(\sum_{j \geq 0} b_{j} X^{j}\right)=0$ if and only if $d_{j}=0$ for all $j \geq 0$. The equation $d_{j}=0$ combrined with the fact that. $p_{0} \circ \theta: M \rightarrow M$ is injective successively yicld $b_{j}=0$ for all $j \geq 0$. Hence $\varphi: M[[X]] \rightarrow M[\{X\}]$ is injective.

(1) $\Rightarrow(3)$. Again the proof is sinnilar to that of $(1) \Rightarrow$ (2) and hence omitted.

Remarks 2.2.

(a) For any $0 \neq M \epsilon A$-mod, the modules $M[X]$ in $A[X]$-rnod and $M[\{X]]$ in $A[[X]]-\bmod$ are never co-Hopfian. In fact the map "Inultiplication by $X$ " is an injective non-sirjective map in both cases.

(b) If $M \epsilon A$-mod is Hopfian we do not know whether $M\left[X, X^{-1}\right]$ will be Hopfian in $A\left[X, X^{-1}\right]$-mod. However if $M\left[X, X^{-1}\right]$ is Hopfian in $A\left[X, X^{-1}\right]$-mod it can be shown that $M$ is Hopfian in $A$-rnod. The proof is sirnilar to the proof (2) $\Rightarrow(1)$ in Theorem 2.1 .

\section{The Commutative case}

Throughout this section unless otherwise stated $A$ denotes a commmtative ring. The following Lemma rnight be woll-known. As we can not find an explicit. reference we include a proof of it hore. 
Lemma 3.1. Let $P$ be an $n \times n$ matrix over $A$. Then there exists $a$ non-zero cohmn vector $\mathbf{a} \epsilon A^{n}$ with $\mathrm{Pa}=0$ if and only if det $P=$ either 0 or a zero divisor in $A$, where 0 denotes the column vector in $A^{\text {rh }}$ with all entries 0 .

Proof: Let $P \mathbf{a}=\mathbf{0}$ with $\mathbf{a} \neq \mathbf{0}$. Let adj $P$ denote the adjugate of $P$. Then $0=(\operatorname{adj} P) P \mathbf{a}=(\operatorname{det} P) I_{n} \mathbf{a}=(\operatorname{det} P) \mathbf{a}$. If $a_{i}$ is a norl-zero entry of a, then $(\operatorname{det} P) a_{i}=0$ with $a_{i} \neq 0$.

Conversely, let $P=d \epsilon A$ and let there exist an element $a \neq 0$ in $A$ with $d a=0$. By induction on $n$ we show that $\exists$ an clement $\mathbf{c} \neq 0$ in $A^{n}$ with $P \mathrm{c}=0$. For $n=1$, we have $P=(d)$ and $P a=0$ with $a \neq 0$ in $A$. Assume the result valid for square matrices of si\%e $\leq n-1$. Let $C_{2, j}$ be the $i, j$ cofactor of $P$. From $P(\operatorname{adj} P) \mathrm{a}=(\operatorname{det} P) \mathrm{a}=\mathbf{0}$ where a is the column vector all of whose entries are $a$ we get. $P\left(\begin{array}{c}C_{11} a \\ \ldots \\ \ldots \\ C_{1 r_{l} a}\end{array}\right)=0$. If $\left(\begin{array}{c}C_{11} a \\ \cdots \\ \cdots \\ C_{1 n} a\end{array}\right) \neq 0$, there is nothing to prove. Otherwise $C_{11} a=0$ and $C_{11}$ is the determinant of the $(n-1) \times(n-1)$ matrix $S$ got from $P$ by deleting the first row and first column. By the inductive assumption 3 an element $\mathbf{v} \neq 0$ in $A^{n-1}$ with $S \mathrm{v}=0$ in $A^{n-1}$. If $\mathrm{c}=\left(\begin{array}{l}0 \\ v\end{array}\right) \in A^{n}$, then c $\neq 0$ ir $A^{n}$ and $P c=0$ in $A^{n t}$.

Theorem 3.2. Let $A$ be a commutative ring with the property that $A$ is co-Hopfian as an $A$-module. Then for each integer $n \geq 1, A^{n}$ is co-Hopfian as an A-module.

Proof: Let $f: A^{n} \rightarrow A^{n}$ be any injective hornomorphisrn of $A$-modules. Let. $P$ denote the matrix of $f$ w.r.t. the standard basis of $A^{n}$. From lemma 3.1 we see that det $P$ is not a zero-divisor in $A$. From remark 1.6(b) we see that $A$ is its own total quotient ring. It follows that $\operatorname{det} P$ is a unit in $A$, hence $P$ is invertible. This means $f$ is an isomorphism.

Examples 3.3. Let $B$ be any abclian group with the property that the $p$-primary torsion $t_{p}(B)$ is not zero for every prime $p$. Let $A=$ $B \oplus \mathcal{Z}$ as an abelian group and let us define multiplication in $A$ by $(b, m)\left(b^{\prime}, m^{\prime}\right)=\left(m b^{\prime}+m^{\prime} b, m m^{\prime}\right) . A$ is a commutative ring. In fact $A$ is the ring got by adjoining an identity elenent to $B$ with the so called zero ring structure on $B$. Every element of the form $(b, m)$ with $m \neq \pm 1$ is a zero-divisor in $A$. In fact if $p$ is any prime divisor of $m$, we can choose an element $0 \neq b^{\prime} \in t_{p}(B)$ with $p b^{\prime}=0$. Then $\left(b^{\prime}, 0\right)(b, m)=(0,0)$. Also 
from $(b, 1)(-b, 1)=(0,1)$ and $(b,-1)(-b,-1)=(0,1)$ we see that all the non-zero divisors in $A$ are invertible. From remark $1.6(\mathrm{~b})$ and theorcm 3.2 we see that $A^{z}$ is co-Hopfian in $A$-mod for every integer $n \geq 1$. $B$ is a two sided ideal in $A$ with $A / B \simeq Z$ as a ring. Thus $B$ is a prime ideal of $A$. However, $B$ is not maximal, becanse $\eta^{-1}(m \mathcal{Z}) \supset B$ for any $m \neq 0$ wherc $\eta: A \rightarrow A / B$ is the canonical quotient map. Thus from remark $1.6(\mathrm{~d})$ we see that there are finitely generated $A$-modules which are not co-Hopfian. In fact the cyclic $A$-module, $A / B \simeq Z$ is itself not co-Hopfian as an $A$-module. For any $m \neq 0$, multiplication by $m$ is an $A$-module homomorphism which is injective but not surjective.

In the above example let us choose $B$ as follows. For some prime po, let $t_{p_{0}}(B)$ be an infinite direct sum of copies of $\mathcal{Z} / p_{0} \mathcal{Z}$ and for primes $p \neq p_{0}$ let $t_{p}(B)$ be any arbitrary $p$-primary torsion abolian group which is not zero. Let $B=$ Dp $_{p}$ t $t_{p}(B)$ where $P$ denotes the set of all primes. Since $B$ is an ideal in $A, B$ is an $A$-submodule of $A$. The $A$-endomorphisrns of $B$ are the samo as the abalian group endomorphisms of $B$. As an abelian group, $t_{p_{0}}(B)$ is neither Hopfian nor co-Hopfian. Since $t_{p o}(B)$ is a direct. summand of $B$, we see that $B$ is neither Hopfan nor 6 -Hoptian as an abelian group and hence as an $A$-module. For any subgroup $H$ of $B$, $H \oplus \mathcal{Z}$ is a subring of $A$. Clearly as an abelian group $B$ does not satisfy the a.c.c. for subgroups. It follows that as a ring, $A$ does not satisfy the a.c.c. for subrings. Since all subgroups of $B$ are ideals in $A$, it is also clear that $A$ is not noctherian. In this example: $A$ is both Hopfian and co-Hopfian as an $A$-module but the submodule $B$ of $A$ is neither Hopfian nor co-Hopfian.

Proposition 3.4. For any commutatine ring $A$ and any intuger $n \geq$ 1 , the ring $M_{r_{n}}(A)$ is Hopfion as an $M_{r_{l}}(A)$-module.

Proof: It suffices to prowe that $M_{n}(A)$ is Hopfian in $M_{n}(A)$-rnod. Let $X$ denote a left zero-divisor in $M_{n}(A)$. We have to show that $X$ is not a left unit. in $M_{r}(A)$. If possible let $Y \in M_{n}(A)$ satisfy $Y X=I_{n}$. Since $A$ is commutative, it follows that $X$ is invertible in $M_{r}(A)$ and hences cannot be a left zero divisor contradicting the original assumption.

Proposition 3.5. Let $A$ be a commutative ring which is co-Hopfunt in $A$-mod. Then $M_{n}(A)$ is co-Hopfian in both the categories $M_{n}(A)$-rood and $\bmod -M_{n}(A)$.

Proof: Let $X \in M_{n}(A)$ be a left non-zero divisor in $M_{n t}(A)$. Then we claim that there exists no non-zcro row vector $\underline{a}=\left(a_{1}, \ldots, a_{n}\right)$ in $A^{n}$ with $\underline{a} X=0\left(0=\right.$ the zero row vector in $\left.A^{n}\right)$. Because if there cxistcd such an $\underline{a}$, then the $n \times n$ matrix $Y$ cach of whose rows is a satisfics 
$Y X=0$ and $Y \neq 0 \in M_{r_{l}}(A)$. From the right analogue of lemma 3.1 we sec that det $X$ is not a zero divisor in $A$. But $A$ being co-Hopfian in $A$-mod, we see that det $X$ is a unit in $A$, hence $X$ is invertible in $M_{n}(A)$. Hence $M_{n}(A)$ is co-Hopfian in $M_{n}(A)$-mod.

The proof for the other half of the proposition is similar.

Let $P$ be a prime idcal in $A$ and $M \epsilon A$-rnod. Let $M_{P}$ be the localization of $M$ regarded as an $A_{P}$-module. A natural query is in what way the Hopfinn (resp. co-Hopfan) nature of $M \in A$-mod related to the Hopfian (resp. co-Hopfian) nature of $M_{p} \in A p$-rnod. The following examples show nothing much can be said.

Examples 3.6.

(a) For any prime $p_{1} \mathcal{Z}_{p_{\infty}} \oplus \mathcal{Z}$ is neither Hopfian nor co-Hopfian in $\mathcal{Z}$-mod. It's localization at the prime ideal 0 of $\mathcal{Z}$ is $\mathcal{Q}$ in $\mathcal{Q}$-mod and $\mathcal{Q}$ is both Hopfian and co-Hoptian in $\mathcal{Q}$-rnod.

(b) For every prime $p$, kt $\mathcal{Z}_{(p)}=\left\{\frac{m}{n} \in Q \mid(n, p)=1\right\}$. Since $\mathcal{Z}_{(p)}$ is noetherian as a ring, $Z_{(p)}$ is Hopfian in $Z_{(p)}$-rnod, honce Hopfian in $\mathcal{Z}$-rnod. If $H=\Theta_{p e P} \mathcal{Z}_{(p)}$, where $P$ is the set of all primes, using the fact that $\operatorname{Hom}_{Z}\left(\mathcal{Z}_{(p)}, \mathcal{Z}_{(q)}\right)=0$ if $p$ and $q$ are distinct primes we see that $H$ is Hopfian in $Z$-mod. Now $Q \otimes H$ is an infinite dircct sum of copies of $\mathcal{Q}$ and hence not Hopfian in $\mathcal{Q}$-mod.

\section{Hopfian and co-Hopfian Boolean rings}

Recall that a ring $A$ is said to be Boolean if $a^{2}=a$ for all $a \epsilon A$. It is well known that any Boolean ring $A$ is conmutative and that $2 a=0$ for any $a \in A$. If $A$ is a Boolean integral domain and $a \neq 0$ in $A$, then from $(a-1) a=0$ we see that $a=1$ and hence $A \simeq \mathcal{Z} / 2 \mathcal{Z}$. In particular it follows that any prime ideal in an arbitrary Boolcan ring $A$ is necessarily maxirnal in $A$. From $1.6(\mathrm{c})$ and (d) we see that all finitely generated modules over $A$ are Hopfian and co-Hopfian. The object of the present section is to determine necsissary and sufficient conditions for $A$ to be Hopfian (resp. co-Hopfian) as a ring using M.H. Stone's representation theorcrn [20]. Given any compact totally disconnected Hausdorff space $X$ let $B(X)$ denote the sct of clopen subsets of $X . B(X)$ turns ont to be a Bookan ring under addition and multiplication defincd by $C+D=C \nabla D$, the symmetric difference of $C$ and $D$, and $C \cdot D=$ $C \cap D$. Let $H$ denote $Z / 2 Z=\{0,1\}$ with the discrete topology. For any sit $S$ let $H^{S}=\prod_{s \in S} H_{s}$ where $H_{s}=H$ for all $s \epsilon S$, endowed with the cartcsian product topology. Given a Boolean ring $A$ ket $X_{A}=\{f \in$ $H^{A} \mid f(a+b)=f(a)+f^{\prime}(b), f(a b)=f(a) f^{\prime}(b)$ and $\left.f(1)=1\right\}$. Then it is known that $X_{A}$ is a closed subspace of $H^{A}$, hence $X_{A}$ is a compact totally disconnected Hausdorff space. Let $T: A \rightarrow B\left(X_{A}\right)$ denote the 
map $T(a)=\left\{f \in X_{A} \mid f(a)=1\right\}$. Then Stone's representation theorem asserts that $T: A \rightarrow B\left(X_{A}\right)$ is a ring isomorphism. A rice account of Stone's represcntation theorem is given in appendix three of [20].

Given any continuous map $\varphi: X \rightarrow Y$ of compact, Hausdorff totally disconnected spaces, for each clopen set $E$ of $Y, \varphi^{-1}(E)$ is a clopen set of $X$; also $B(\varphi): B(Y) \rightarrow B(X)$ defined by $B(\varphi)(E)=\varphi^{-1}(E) \in B(X)$ for each $E \in B(Y)$ is easily seen to be a ring homomorphism.

Conversely, given a homomorphism $x: A \rightarrow B$ of Boolean rings there is an associated map $X(a): X_{B} \rightarrow X_{A}$. For defining this observe that $X_{B}$ is nothing but the set of ring homomorphisms of $B$ into $Z / 2 \mathcal{Z}$, regarded as a topological subspace of $H^{l}$. Given any ring homomorphism $f: B \rightarrow$ $\mathcal{Z} / 2 \mathcal{Z}$, clearly $f \circ \alpha: A \rightarrow \mathcal{Z} / 2 \mathcal{Z}$ is a ring homomorphism. The map $X(\alpha)$ is given by $X(\alpha)(f)=f \circ \alpha$. It turns out to be continuous. Let $B(X(\alpha)): B\left(X_{A}\right) \rightarrow B\left(X_{B}\right)$ be the ring homomorphism associated to $X(\alpha)$ as described in the carlicr paragraph. Then

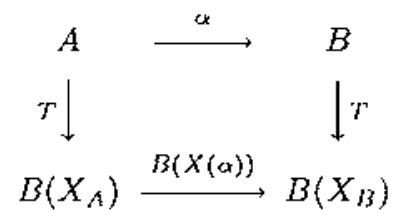

diagram 4.1

is known to be a commutative diagram. Actually, for any $a \in A$, we have $T \circ \alpha(a)=\{f: B \rightarrow Z / 2 Z \mid f$ a ring homomorphism with $f(\alpha(a))=1\}$ and $B(X(\alpha)) \circ T(a)=B(X(\alpha))\{g: A \rightarrow \mathcal{Z} / 2 \mathcal{Z} \mid g$ a ring homomorphism satisfying $g(a)=1\}=X(a)^{-1}\{g: A \rightarrow Z / 2 Z \mid g$ a ring homomorphism satisfying $g(a)=1\}=\{f: B \rightarrow \mathcal{Z} / 2 \mathcal{Z} \mid f$ a ring homonorphism with $(X(\alpha)(f))(a)=1\}=\{f: B \rightarrow \mathcal{Z} / 2 Z \mid f$ a ring homomorphism with $f(\alpha(a))=1\}=T \circ \alpha(a)$. This proves the commutativity of diagram 4.1.

Lemma 4.1. Let $X$ be a compact Hausdorff totally disconnected space and $Y$ a closed subspace of $X$. Let $F$ be any clopen subset of $Y$. Then there exists a clopen subset $C$ of $X$ with $C \cap Y=F$.

This is actually lemma \& in Hiremath's paper [12].

Proposition 4.2. Let $\alpha: A \rightarrow B$ be a homomorphism of Boolean rings. Then

(i) $\alpha$ is surjective $\Leftrightarrow X(\alpha): X_{B} \rightarrow X_{A}$ is injective $\Leftrightarrow B(X(\alpha))$ : $B\left(X_{A}\right) \rightarrow B\left(X_{B}\right)$ is surjective.

(ii) $\alpha$ is injective $\Leftrightarrow X(\alpha): X_{B} \rightarrow X_{A}$ is surjective $\Leftrightarrow B(X(\alpha))$ : $B\left(X_{A}\right) \rightarrow B\left(X_{B}\right)$ is injective. 
Proof: (i) From the commutativity of diagram 4.1, we sce that $\alpha$ is surjective $\Leftrightarrow B(X(\alpha)): B\left(X_{A}\right) \rightarrow B\left(X_{B}\right)$ is surjective.

Assume $\alpha$ surjective. If possible let $X(\alpha)$ be not injective. Let $u \neq v$ be elements in $X_{B}$ with $X(\alpha)(u)=X(\alpha)(v)$. There exist clopen sets $E, F$ in $X_{B}$ with $u \in E, v \in F$ and $E \cap F=\phi$. Since $B(X(\alpha))$ is surjective, there exist clopen sets $C, D$ in $X_{A}$ satisfying $X(\alpha)^{-1}(C)=E$ and $X(\alpha)^{-1}(D)=F$. From $u \in E$ and $v \in F$ we get $X(\alpha)(u) \in$ $C, X(\alpha)(v) \in D$. But we have $X(\alpha)(u)=X(\alpha)(v)=t$ (say). Then $t \in C \cap D$ and hence $u$ and $v$ are in $X(\alpha)^{-1}(C \cap D)=E \cap F$. This contradicts the fact that $E \cap F=\phi$. This proves the implication $\alpha$ surjective $\Rightarrow X(\alpha)$ injective.

Conversely, assume $X(\alpha): X_{B} \rightarrow X_{A}$ injective. We will prove that $B(X(\alpha)): B\left(X_{A}\right) \rightarrow B\left(X_{B}\right)$ is surjective. Since $X(\alpha): X_{B} \rightarrow X_{A}$ is injectivc and the spaces involved are compact Hausdorff, it follows that $X(\alpha)$ : $X_{B} \rightarrow X(\alpha)\left(X_{B}\right)$ is a homeomorphism when we regard $X(\alpha)\left(X_{B}\right)$ as a subspace of $X_{A}$. Given any clopen subsct $F$ in $X_{B}$, from lemma 4.1 we see that there exists a clopen subset, $C$ of $X_{A}$ with $C \cap X(\alpha)\left(X_{B}\right)=$ $X(\alpha)(F)$, or equivalently $X(\alpha)^{-1}(C)=F$. Thus $B(X(\alpha)): B\left(X_{A}\right) \rightarrow$ $B\left(X_{B}\right)$ is surjective, completing the proof of (i).

(ii) Again from the commutativity of diagram 4.1 we see that $\alpha$ is injective $\Leftrightarrow B(X(\alpha)): B\left(X_{A}\right) \rightarrow B\left(X_{B}\right)$ is injective.

Assume $\alpha$ injective. If possible let $X(\alpha): X_{B} \rightarrow X_{A}$ be not surjective. Then there cxists sorne $x \in X_{A}$ with $x \notin X_{A}-X(\alpha)\left(X_{B}\right)$. Since $X_{A}-X(\alpha)\left(X_{B}\right)$ is arn open set in $X_{A}$ containing $x$ and since clopen neighbourhoods form a fundamental system of neighbourhoods of any point in $X_{A}$, we get a clopen set $C$ in $X_{A}$ with $x \in C \subset X_{A}-X(\alpha)\left(X_{B}\right)$. The sets $X_{A}$ and $X_{A}-C$ are distinct clopen sets in $X_{A}$ with $X(\alpha)^{-1}\left(X_{A}\right)=$ $X(\alpha)^{-1}\left(X_{A}-C\right)=X_{B}$ showing that $B(X(\alpha))$ is not injective. This contradicts the assumption that $\alpha$ is injective.

Conversely, assume that $X(\alpha): X_{B} \rightarrow X_{A}$ is surjective. If $\varphi: S \rightarrow T$ is any set theoretic surjection and $T_{1} \neq T_{2}$ arc distinct subsets of $T$, it is clear that $\varphi^{-1}\left(T_{1}\right) \neq \varphi^{-1}\left(T_{2}\right)$. In particular if $C, D$ are distinct clopen subsets of $X_{A}$ we see that $X(\alpha)^{-1}(C) \neq X(\alpha)^{-1}(D)$, showing that $B(X(\alpha)): B\left(X_{A}\right) \rightarrow B\left(X_{B}\right)$ is injective. This in turn shows that $\alpha: A \rightarrow B$ is injective, thus completing the proof of (ii).

Proposition 4.2 implies the well-known result that the isomorphism type of the ring $A$ determines the homeomorphism type of the space $X_{A}$. It is well-known [21] that $f \rightarrow$ Ker $f$ establishes a bijection between points of $X_{A}$ and miximal ideals of $A$. We can transport the topology of $X_{A}$ to max Spec $A$ using the above bijection. If we start with a compact Hausdorff totally disconnected space $X$, for each $x \in X$ if we 
set $I_{x}=\{C \in B(X) \mid x \notin C\}$ then $x \mapsto I_{x}$ is a hoeomorphism of $X$ with $\max \operatorname{Spcc} B(X)$.

Definition 4.3. A topological space $X$ is said to be Hopfian (resp. co-Hopfian) in the category Top if every surjective (resp. injective) continuous rap $f: X \rightarrow X$ is a homeomorphism.

The main result of this section is the following.

Theorem 4.4. A Boolean ring $A$ is Hopfian (resp. co-Hopfan) as a ring if and only if $X_{A}$ is co-Hopfian (resp. Hopfian) in the category Top.

Proof: Immediate consequence of proposition 4.2.

Remarks 4.5. Let $J$ be any finite set. The product space $H^{J}$ where $H=\{0,1\}$ is neither Hopfian nor co-Hopfian. As a set $H^{J}$ is the set of all maps of $J$ into $H$. For any sct theoretic map $\theta: J \rightarrow J$ we have an induced map $f \mapsto f \circ \theta$ of $H^{J}$ into $H^{J}$, which is easily seen to be continuous. If $\theta$ is an injective (resp. surjective) map which is not a bijection, then $f \mapsto f \circ \theta$ is a surjective (resp. injective) map which is not bijective.

Since $A=B\left(H^{J}\right)$ is a commutative ring in which cvery prime ideal is rnaximal, all $f \cdot g A$-modulcs are simultaneously Hopfian and co-Hopfiar in the category of $A$-modulcs but $A$ is neither Hopfian nor co-Hopfian as a ring.

It would be nice to charactcrize completely the Hopfian (resp. coHopfian) compact Hausdorff totally disconnected spaccs.

\section{Hopfian and co-Hopfian function algebras}

Let $K$ be a commutative ring and $K$-alg denote the eategory of $K$ algebras.

Definition 5.1. A $K$-algebra $A$ is said to be Hopfian (resp. coHopfan) as a $K$-algebra if any surjective (resp. injective) $K$-algebra homomorphism $f: A \rightarrow A$ is isomorphism.

Let $\mathcal{R}$ (resp. $\mathcal{C}$ ) denote the field of real (resp. complex) numbers with the usual topology. For any compact Hausdorff space $X$ let $C_{\mathcal{R}}(X)$ (resp. $C_{C}(X)$ ) denote the $\mathcal{R}$ (resp. $\mathcal{C}$ )-algcbra of continuous functions from $X$ to $\mathcal{R}$ (resp. $\mathcal{C}$ ). Using the Gelfand respresentation theorem we will determine necessary and sufficient conditions for $C_{\mathcal{R}}(X)$ (resp. $C_{\mathcal{C}}(X)$ ) to be Hopfian or co-Hopfian in the category $\mathcal{R}$-alg (rcsp. $\mathcal{C}$-alg). We will mainly concentrate on $C_{R}(X)$. Similar results are valid for $C_{\mathcal{C}}(X)$.

$X$ denotes a compact Hausdorff space and $C(X)$ denotes the $\mathcal{R}$-algebra $C_{\mathcal{R}}(X)$. It is well-known that the map $x \mapsto(f(x))_{f \in C(X)}$ is a topological 
imbedding of $X$ into $\Pi_{f \in C(X)} \mathcal{R}_{f}$ with the cartesian product topology, wherc $\mathcal{R}_{f}=\mathcal{R}$ for each $f \in C(X)$. Also $x \mapsto m_{x}=\{f \in C(X) \mid f(x)=$ $0\}$ is a bijection from $X$ to the set of maximal deals in the $\mathcal{R}$-algebra $C(X)$. If $X \stackrel{\varphi}{\rightarrow} Y$ is a continuous map of compact Hausdorff spaces, there is an induced homomorphism $\varphi^{*}: C(Y) \rightarrow C(X)$ in $\mathcal{R}$-alg given by $\varphi^{*}(g)=g \circ \varphi$ for every $g \in C(Y)$. Also given any $\mathcal{R}$-algcbra homomorphism $\alpha: C(Y) \rightarrow C(X)$, there is a unique continuous map $p: X \rightarrow Y$ satisfying $\alpha=\varphi^{*}$. To see this, for any $x \in X, \alpha^{-1}\left(m_{x}\right)$ is a maximal ideal of $C(Y)$ and hence $\alpha^{-1}\left(m_{x}\right)=m_{\varphi(x)}$ for a unique element $\varphi(x) \in Y$. If $j_{X}: X \rightarrow \Pi_{f \in C(X)} \mathcal{R}_{f}$ and $j_{Y}: Y \rightarrow \Pi_{g} \in C(Y) \mathcal{R}_{g}$ denote the imbeddings $j X(x)=(f(x))_{f \in C(X)}$ and $j_{Y}(y)=(g(y))_{g \in C(Y) Y}$ respectively, then the set theoretic map $\varphi: X \rightarrow Y$ obtained above satisfies the condition that.

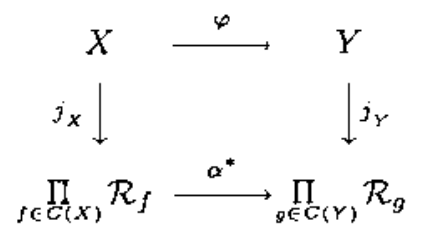

diagram 5.2

is commutative, where $\alpha^{*}\left(\left(r_{f}\right)_{f \in C(X)}\right)=\left(s_{g}\right)_{g \in C(Y)}$ with $s_{g}=r_{\alpha(g)}$. Since $\alpha^{*}$ composed with any projection $\Pi \mathcal{R}_{g} \rightarrow \mathcal{R}_{g}$ is continuous we see that $\alpha^{*}$ is continuous, hence $\varphi$ is continuous, provided we check the commutativity of the diagram 5.2. But it is straight forward. Thus the set of $\mathcal{R}$-algetbra homomorphisms $C(Y) \rightarrow C(X)$ is the same as the set $\left\{\varphi^{*}:\{\varphi: X \rightarrow Y\right.$ continuous $\}$. The results quoted so far are well-known ([20, pages 327-330]) .

Proposition 5.2. Let $\varphi: X \rightarrow Y$ be a continuous map of compact Hausdorff spaces. Then

(i) $\varphi^{*}: C(Y) \rightarrow C(X)$ is injective $\Leftrightarrow \varphi: X \rightarrow Y$ is surjective.

(ii) $\varphi^{*}: C(Y) \rightarrow C(X)$ is surjective $\Leftrightarrow \varphi: X \rightarrow Y$ is injective.

Proof:

(i) Suppose $\varphi: X \rightarrow Y$ is not surjective. Then $\varphi(X)$ is a proper elosed subsct of $Y$. We can pick an element $b \in Y-\varphi(X)$. Let $h: \varphi(X) \rightarrow \mathcal{R}$ be any continuous function. Then we can get continuous extensions $g_{1}: Y \rightarrow \mathcal{R}, g_{2}: Y \rightarrow \mathcal{R}$ of $h$ with $g_{1}(b)=0$ and $g_{2}(b)=1$ (by Tietze extcnsion theorem). Then $g_{1} \neq g_{2}$ in $C(Y)$ but $\varphi^{*}\left(g_{1}\right)=h \circ \varphi=\varphi^{*}\left(g_{2}\right)$ since $g_{1}\left|\varphi(X)=g_{2}\right| \varphi(X)=h$. Thus $\varphi$ not surjective $\Rightarrow \varphi^{*}$ not injective or equivalently $\varphi^{*}$ injective $\Rightarrow \varphi$ surjective. 
If $\varphi: X \rightarrow Y$ is surjective, then for any two set theoretic maps $g_{1}: Y \rightarrow$ $\mathcal{R}, g_{2}: Y \rightarrow \mathcal{R}$, we have the implication $g_{1} \circ \varphi=g_{2} \circ \varphi \Rightarrow g_{1}=g_{2}$. In particular this implication is true with $g_{1}, g_{2}$ in $C(Y)$. This proves (i). (ii) Suppose $\varphi$ is not injective, say $x_{1} \neq x_{2}$ in $X$ satisfy $\varphi\left(x_{1}\right)=$ $\varphi\left(x_{2}\right)$. Any $f \in C(X)$ of the form $g \circ \varphi$ with $g \in C(Y)$ has to satisfy $f\left(x_{1}\right)=f\left(x_{2}\right)$. However, we do know that $\exists f \in C(X)$ with $f\left(x_{1}\right)=0$ and $f\left(x_{2}\right)=1$. Thus $\varphi^{*}: C(Y) \rightarrow C(X)$ is not surjective.

Conversely, assurne that $\varphi$ is irjective. Then $\varphi: X \rightarrow \varphi(X)$ is a horreomorphism arid $\varphi(X)$ is closed in $Y$. Given ary $f \in C(X), h: \varphi(X) \rightarrow \mathcal{R}$ defined by $h \varphi(x)=f(x)$ is continuous. By Tictze extension theorcm, there cxists $g \in C(Y)$ with $g \mid \varphi(X)=h$. Then $\varphi^{*}(g)=f$, showing that. $\varphi^{*}: C(Y) \rightarrow C(X)$ is surjective.

Theorem 5.3. Let $X$ be a compact Hausdorff space. Then $C(X)$ is Hopfian (resp. co-Honfian) as an $\mathcal{R}$-algebra if and only if $X$ is co-Hopfiar (resp. Hopfian) as a topological space.

Proof: Immediate consequence of proposition 5.2.

\section{Hopfian and co-Hopfian objects in Top among compact manifolds}

For each integer $n \geq 1$ let $D^{n}$ denote an $n$-disk. Wo may take $D^{n}=$ $\left\{x \in \mathcal{R}^{n} \mid\|x\| \leq 1\right\}$ where $\|x\|$ denotes the usual norm in $\mathcal{R}^{n}$. By definition $D^{0}$ consists of a point. For $n \geq 1$, the maps $x \mapsto \frac{1}{2} x$ is a continuous injection which is not a surjection. The map $\theta: D^{n} \rightarrow D^{n}$ given by

$$
\theta(x)= \begin{cases}2 x & \text { for }\|x\| \leq \frac{1}{2} \\ \frac{x}{\|x\|} & \text { for }\|x\| \geq \frac{1}{2}\end{cases}
$$

is a continuous surjection which is not injective. Thus $D^{n}$ is neither Hopfian nor co-Hopfian for $n \geq 1$. Observe that $\theta: D^{n} \rightarrow D^{n}$ defined above has the additional property that $\theta \mid S^{n-1}=I d_{s^{n-1}}$. Let, $M^{n}$ be any compact topological manifold (with or without boundary) of dimension $n \geq 1$. Then imbedding a disk $D^{n}$ in $M^{n}$ we can defire a continuous surjection $f: M^{n} \rightarrow M^{n t}$ with $f \mid\left(M^{n}-\operatorname{Int} D^{n t}\right)=I d_{\left(M^{n}-\Gamma_{n i} D^{n}\right)}$ and $f \mid D^{r t}$ a continuous surjection of $D^{n_{k}}$ with itself satisfying $f \mid S^{r-1}=I d_{s^{n-1}}$ and $f \mid D^{n}$ not injective. It follows that $M^{n}$ is not Hopfann. Thus we obtain the following. 
Proposition 6.1. The only compoct manifolds (with or without) boundary which are Hopfian are finite discrete spoces.

As usual for any topological space $X$ we denote the set of arcwise connected components of $X$ by $\Pi_{0}(X)$.

Theorem 6.2. Let $M^{n}$ and $N^{n}$ be a compact topological manifolds of the same dimension $n \geq 1$, both of them unithout boundary. Suppose further $\left|\Pi_{o}\left(M^{n}\right)\right|=\left|\Pi^{0}\left(N^{n}\right)\right|$. Then any continuous injection $f: M \rightarrow N$ is a homeomorphism.

Proof: Since $M^{n}$ and $N^{n}$ are compact we see that $\left|\Pi_{0}\left(M^{n}\right)\right|=\left|\Pi_{0}\left(N^{n}\right)\right|$ $<\infty$. Let $\left\{M_{i}^{n}\right\}_{i=1}^{k}$ denote the set of connected components of $M^{n}$. Each $M_{i}^{n}$ is a compact connected manifold without boundary, of dimension $n$. Hence $f\left(M_{i}^{n}\right)$ is a compact, connected subset of $N^{n}$. Since $f$ is injective we sec that $f \mid M_{i}^{n}: M_{i}^{n} \rightarrow f\left(M_{i}^{n}\right)$ is a homemorphism. By invariance of dornain it follows that $f\left(M_{i}^{n^{2}}\right)$ is open in $N^{n}$. Thus $f\left(M_{i}^{n}\right)$ is open and closed in $N^{n}$ and also connected. Hence $f\left(M_{i}^{n}\right)$ is a connected component. of $N^{n}$. From the injectivity of $f$ it follows that if $i \neq j, f\left(M_{i}^{n}\right)$ and $f\left(M_{j}^{n}\right)$ are distinct connected components of $N^{n}$. Since $\left|\Pi_{0}\left(N^{n}\right)\right|=\left|\Pi_{0}\left(M^{n}\right)\right|=$ $k<\infty$, it follows that $\left\{f\left(M_{i}^{n}\right)\right\}_{i=1}^{k}$ are all the connected components of $N^{n}$, hence $f: M^{n} \rightarrow N^{n}$ is into. From the compact Hausdorff nature of $M$ and $N$ we see that $f: M^{n} \rightarrow N^{n}$ is a homeormorphisini

As an immediate consequence of theoron 6.2 we get

Corollary 6.3. Any compact manifold $M^{n}$ unthout boundary is coHopfan in Top.

Proposition 6.4. Any compact manifold $M^{n}$ with a non-empty boundary $\partial M$ is never co-Hopfian in Top.

Proof: By Morton Brown's collaring theorem, there exists a homeomorphism $\theta: \partial M \times[0,1] \rightarrow W$ where $W$ is a neighbourhood of $\partial M$ in $M$, satisfying $\theta(x, 0)=x$ for all $x \in \partial M$. Let $f: M \rightarrow M$ be defined by $f(u)=u$ for all $u \in M-\theta(\partial M \times[0,1)), f(\theta(x, t))=\theta\left(x, \frac{1+t}{2}\right)$ for all $x \in \partial M$ and $t \in[0,1]$. Then $f: M \rightarrow M$ is a continuous injection which is not a surjection.

Let $T o p^{2}$ denote the catcgory of pairs of topological spaces.

Definition 6.5. A pair $(X, A) \in T o p^{2}$ is called Hopfian (resp. coHopfian) if any surjective (resp. injective) map $f:(X, A) \rightarrow(X, A)$ of pairs is a homeomorphism.

For any space $X$ let $H_{i}(X)$ denote the singular hornology with integer coefficients. 
Theorem 6.6. Let $M, N$ be compact manifolds with boutndary satisfying the following conditions.

(i) $\operatorname{dim} M=\operatorname{dim} N$

(ii) rank $H_{0}(\partial M)=\operatorname{rank} H_{0}(\partial M)$, rank $H_{0}(M)=\operatorname{rank} H_{0}(N)$ and $\operatorname{rank} H_{0}(M, \partial M)=\operatorname{rank} H_{0}(N, \partial N)$.

Then any injective continuous map $f:(M, \partial M) \rightarrow(N, \partial N)$ is a homcomorphism.

Proof: Let. $V$ denote the double $M_{+} \cup_{\partial M} M_{-}$of $M$. Let $r=$ rank $H_{0}(M)$ and $s=\operatorname{rank} H_{0}(M, \partial M)$. If $i: \partial M \rightarrow M$ denotes the inclusion, then from the exact sequence $H_{0}(\partial M) \stackrel{i^{*}}{\rightarrow} H_{0}(M) \rightarrow H_{0}(M, \partial M) \rightarrow 0$ we see that rank In $i_{*}=r-s$. From the Mayer-Vietoris sequence $H_{0}(\partial M) \stackrel{\left(i_{+}\right) .\left(i_{-}\right)}{\longrightarrow} H_{0}\left(M_{+}\right) \oplus H_{0}\left(M_{-}\right) \rightarrow H_{0}(V) \rightarrow 0$ where $i_{+}: \partial M \rightarrow$ $M_{+}, i_{-}: \partial M \rightarrow M_{-}$are the respective inclusions, we sce that rark $H_{0}(V)$ $=2 r-$ rank of image $\left(\left(i_{+}\right)_{*},\left(i_{-}\right)_{*}\right)$. However $\operatorname{Im}\left(\left(i_{+}\right)_{*},\left(i_{-}\right)_{*}\right)$ is the same as the diagonal subgroup of $\operatorname{Im} i_{*} \oplus \operatorname{Im} i_{*}$, hence has the same rank as Im $i_{*}$. Thus rank $H_{0}(V)=2 r-(r-s)=r+s$. Similarly if $W$ denotes the doulbe $N_{+} \cup_{9} N N_{-}$we have rank $H_{0}(W)=r+s$. In particular we get $\left|\pi_{0}(\partial M)\right|=$ rank $H_{0}(\partial M)=$ rank $H_{0}(\partial N)=\left|\pi_{0}(\partial N)\right|$ and $\left|\pi_{0}(V)\right|=r+s=\left|\pi_{0}(W)\right|$.

$f \mid \partial M: \partial M \rightarrow \partial N$ is an injective continuons map and $\left|\pi_{0}(\partial M)\right|=$ $\left|\pi_{0}(\partial N)\right|$. Hence theorem 6.2 implies that $f \mid \partial M$ is a honeonomphism. There is a well-dofined contimous map $g: V \rightarrow W$ satisfying $g \mid M_{+}: M_{+} \rightarrow$ $N_{+}$and $g \mid M_{-}: M_{-} \rightarrow N_{-}$are the same as $f$. Then $g$ is injective and $\left|\pi_{0}(V)\right|=\left|\pi_{0}(W)\right|$. From theorem 6.2 aggain we see that $g: V \rightarrow W$ is a homeomorphism. It follows immediately that $f:(M, \partial M) \rightarrow(N, \partial N)$ is a homeomorphism.

Corollary 6.7. If $M$ is any compact monifold with boundary $\partial M$ then $(M, \partial M)$ is a co-Hopfion olject in Top ${ }^{2}$.

Proof: Immediate consequence of theorem 6.6 .

\section{Theorem 6.8 .}

(i) If $M$ is any compact manifold unthout boundary then $C(M)$ is Hopfian in $\mathcal{R}$-alg.

(ii) If $M$ is any compact topological monifold with a zom-snpty boundary $\partial M$, then $C(M)$ is reither Hopfan nor co-Hopfian in $\mathcal{R}$-allg.

(iii) If $M$ is a compact manifold, then $C(M)$ is co-Hopfian in $\mathcal{R}$-all if and only if $M$ is a finite set. 
Proof: Tmmediate consequence of theorem 5.3, corollary 6.3 and propositions 6.1 and 6.4 .

\section{Some related results and counter examples}

Recill that, a ring $A$ is said to be left (resp. right) $\pi$-regular if given any $a \epsilon A$ there exists in element $b \in A$ and an integer $n \geq 1$ satisfying $a^{n}=b a^{n+1}$ (resp. $a^{n}=a^{n+1} b$ ). F. Dischinger [7], [8] has shown that $\pi-$ regularity is left right symmetric. Before Dischinger obtained this result, G. Azurnaya [3] referred to a ring which is both left and right $\pi$-regular as a strongly $\pi$-regular ring. By Dischinger's result. $A$ is left $\pi$-regular if and only if $A$ is right $\pi$-regular if and only if $A$ is strongly $\pi$-regular. In [7], [8] Dischinger also obtained the following results:

1. A ring $A$ is strongly $\pi$-regular if and only if every cyclic left or right. A-module is co-Hopfian.

2. For a ring $A$ the following conditions are equivalent.

(i) Every finitely gencrated left $A$-module is co-Hopfian.

(ii) Evcry finitely generated right $A$-nodule is co-Hopfinn.

(iii) $M_{n}(A)$ is strongly $\pi$-rcgular for all integers $n_{t} \geq 1$.

In [9] K.R. Goodearl introduced the concept of a left repetitive ring. A ring $A$ is said to be left repetitive if given any $a \in A$ and any $f \cdot g$ left idcal $I$ of $A$, the left ideal $\sum_{n_{2} \geq 0} I a^{n}$ is $f \cdot g$. One of the results proved by Goodearl in [9] is the following:

3. Every $f \cdot g M \in A$-mod is Hopfian if and only if $M_{n}(A)$ is left repetitive for all integers $n \geq 1$.

A good report on these questions inchuding now proots and rew results can be found in [13].

Examples 7.1.

(a) It is clear that $M \in A$-nod Hopfinn $\Rightarrow$ End $(A M)$ directly finite. In [19] J.C. Stepherdson gives examples of directly finite $A$ with $M_{n}(A)$ not directly finits for some integer $n \geq 2$. For any such ring $A$, we have $A$ Hopfian in $A$-rnod. Also $M_{r}(A)$ is not Hopfian in $M_{u}(A)$-rnod. Since $A^{n}$ is a direct summand of $M_{k}(A)$ in $A$ mod, whenever $k^{2}>n$, we also see that $M_{k}(A)$ is not hopfan in A-mod whenever $k^{2}>n$.

(b) In part C of [5] G.M. Bergman constructs for each integer $n>$ I a ring $A$ with the property that all rogular elonents in $A$ are invertible, but $M_{n}(A)$ is not its own classical ring of quotients. In [13] P. Menal constructs a ring $A$ which is its own classical quotient ring but $M_{r_{2}}(A)$ is not Ore, hence $M_{n}(A)$ does not even have a classical ring of quotients. A careful inspection shows that in both 
thesc examples the left regular and the right regular elements of $A$ coincide. Hence by proposition 1.4 in our present paper $A$ is coHopfian in both $A$-mod and mod- $A$. However, $M_{n}(A)$ is ncither co-Hopfian in $M_{r_{t}}(A)$-mod nor co-Hopfian in mod- $M_{r}(A)$.

(c) In section 5 of [16] it is remarked that W.L. May has a method of obtaining an infinite abelian Hopfan group $G$ such that the complex group algebra $\mathcal{C}(G)$ is not Hopfian as a $\mathcal{C}$-algebra, hence not Hopfian as a ring. In this example $\mathcal{C}$ is Hopfian as a ring, $G$ is Hopfian as a group but $C[G]$ is not Hopfian as a ring.

\section{Open problems}

1. If $A$ is Hopfian as a ring, is $A[X]$ Hopfian as a ring?

2. If $A$ is co-Hopfian as is ring and $G$ a co-Hopfiar group is $A \mid G]$ co-Hopfian as a ring?

3. If $A$ is Hopfian in $A$-mod and $G$ a Hopfian group is $A[G]$ Hopfann in $A[G]$-mod?

4. If $A$ is co-Hopfian in $A$-mod and $G$ a co-Hopfian group is $A[G]$ co-Hopfian in $A[G]$-mod?

5. If $A$ is Hopfian (resp. co-Hopfian) as a ring is it true that $M_{n}(A)$ is Hopfiarn (resp. co-Hopfian) as a ring?

6. Characterize the Hopfian (resp. co-Hopfian) objects in Top among compact Hausdorff totally disconnected spaces.

7. If $M \in A$-mod is Hopfian is $M\left[X, X^{-1}\right]$ Hopfian in $A\left[X, X^{-1}\right]$ mod?

\section{References}

1. F.W. ANDERson AND K.R. Fuller, "Rings and Categoties of Modules," Graduate Texts in Mathematics 13, Springer-Verlag, Now York, 1973.

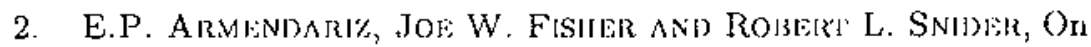
injective and surjective endomorphisms of finitely gencrated modules, Communications in Algebra 6 (1978), 659-672.

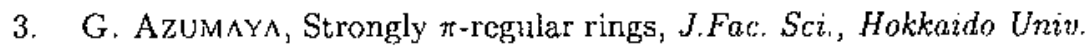
13 (1954), 34-39.

4. G. BAUMSLAG, "Topics in Abelian Groups," Edited by J. Irwin and E.A. Walkcr, Scott Foresmann and Company, 1963, pp. 331335.

5. G.M. Blskgman, Some examples in PI Ring Thoory, Istael I Mafh. 18 (1974), 257-277. 
6. A.W. Cilutrers and C.R. Hajarnavis, "Rings with chain conditions," Research Notes in Mathomaties 44, Pitrnan Publishing Limited, 1980.

7. F. Discuinger, Sur lcs anncaux foretement $\pi$-reguliers, C.R. Acad. Sci. Paris, Ser. A 283 (1976), 571-573.

8. F. Disclinger, Stark $\pi$-regular Ringe, Dissertation, Ludwig-Maximilians-Universitat, Munchen, 1977.

9. K.R. GoodEari, Surjective endomorphisms of finitely generated modules, Comm. in Algebra 15 (1987), 589-609.

10. P.J. HizTON AND J. ROITBERG, Relative epimorphisms and monomorphisms in homotopy theorcy, Composition Mathematica 61 (1987), 353-367.

11. Y. Hiraxo, On Filting's Lemma, Hiroshima Moth. J. 9 (1979), $623-626$.

12. V.A. Hinemath, Hopfian Rings and Hopfian Modules, Indian J. Pure and Appl. Math. 17 (1986), 895-900.

13. P. Mrnal, Cancellation modules over regular rings, Proceedings in ring Theory, Granada, 1986 SLNM 1328.

14. P. Menal, Morita equivalence and quotient rings, Results in Math. 13 (1988), 137 139.

15. M. ORZECH, Onto endomorphisms are isomorphisms, A7ner. Math. Monthly 78 (1971), 357-362.

I6. M. OrzeCH AND L. RiBes, Residual finiteness and the Hopf property in Rings, J. Alg. 15 (1970), 81-88.

17. P. RiBenborm, "Rings and Modules," Tracts in Mathematics 24, Interscicncc Publishers, 1969.

18. J. RolTrerg, Residually finite Hopfian and co-Hophan spaces, Contemp. Math. 37 (1985), 131-144.

19. J.C. Steptiendson, Inverses and zero divisors in matrix rings, Proc. London Math. Soc. (1951), 71-85.

20. G.F. Simmons, "Introduction to Topology and Modern Anolysis," McGraw-Hill Book Company, 1963.

21. M.H. SrON $\mathrm{E}_{3}$ Applications of the theory of Boolean Rings to General Topology, Trans. A.M.S. 41 (1937), 375-481.

22. J.R. STrookrR, Lifting projectives, Nagoya Math. J. 27 (1966), $747-751$.

23. K. VARADARA.JAN, A generalization of Hilbert's Basis Theorem, Communications in Algebra 10 (1982), 2191 2204. 
24. W. Vasconceros, On finitely generated flat modules, Trans. A.M.S. 138 (1969), 505-512.

25. W. VASCONCELOS, Injective endomorphisms of finitely generated modules, Proc. A.M.S. 25 (1970), 900-901.

The University of Calgary

Calgary, Alberta

CANADA T2N $1 \mathrm{NA}$

Rebut el 6 de Febrer de 1992 